\title{
Manfred Körber
}

\section{Sozialethische Notizen zu Digitalisierung - Bildung - Globale Gerechtigkeit}

\begin{abstract}
Zusammenfassung
Der Beitrag beschreibt Aspekte des Zusammenhangs von Globalisierung, Digitalisierung und Bildung. Er nimmt die Perspektive einer handlungsorientierten Sozialethik ein und skizziert unter den Slogans aufklären - erzählen - vernetzten befähigen das Programm einer globalen Bildungsanstrengung als politische Praxis.
\end{abstract}

Schlüsselworte: Digitalisierung, Globalisierung, Bildung

\begin{abstract}
The contribution explains aspects of the relation between globalisation, digitalisation and education. It takes the perspective of an action-oriented social ethic and designs the program of global educational efforts as political practice with the slogan explain - tell - reticulate - empower.
\end{abstract}

Keywords: Digitalisation, Globalisation, Education

\section{Einleitung}

Viel wird über die Auswirkungen der Digitalisierung diskutiert. Es scheint so, als würden sich die Lebensbedingungen der Menschen durch den technologischen Wandel grundlegend ändern. Und immer, wenn sich die Lebensbedingungen von Menschen radikal verändern, wird gerne nach der Ethik gerufen. Das Interesse an der Herbeigerufenen ist dabei sehr unterschiedlich - mal soll sie normative Grundlagen sichern, mal gesellschaftliche oder wirtschaftliche Entwicklungen legitimieren, mal soll sie kritisch sein. So ist es auch mit der Ethik im Prozess der Digitalisierung.

Ethische Reflexion ist allerdings nie neutral, auch wenn sie es versucht, sie hat ihren Standort und ihr Interesse. Sie begibt sich in den Dienst von gesellschaftlichen und wirtschaftlichen Kräften. So reflektiert beispielsweise eine unternehmensbezogene digitale Ethik über „Big Data“ anders als eine den Bürgerrechtsfragen nahestehende. Sie mögen sich in Analyse und normativen Begründungen noch nahe sein, aber spätestens bei den Forderungen und Empfehlungen kommt es zu entgegengesetzten Positionen. Ethik ist somit immer Teil gesellschaftlicher Aushandlungsprozesse - das ist ihre Macht und Ohnmacht zugleich.

Mit Blick auf die Digitalisierung steht die ethische Reflexion noch am Beginn. Seit etwa 2014 gibt es vermehrt Gründungen von Einrichtungen und Initiativen, so etwa das „Institut für Digitale Ethik“ in Stuttgart oder die Gruppe „D21“. Große Stiftungen nehmen sich der Thematik an und seit 2016 mangelt es nicht an „Digitalen Manifesten“ von Industrie und Politik sowie an interdisziplinären Forschungsprojekten.

Der Fokus dieser Initiativen ist meist auf die nationalen Herausforderungen im Zuge der Transformationsprozesse, die durch die Digitalisierung ausgelöst werden, gerichtet. Internationale, interkulturelle oder entwicklungspolitische Kontexte spielen nur am Rande eine Roll.

Der hier vorgelegte Beitrag erhebt, angesichts der Größe der Fragestellung, nicht den Anspruch, die vielfältigen Aspekte des Zusammenhangs von Globalisierung, Digitalisierung und Bildung in sozialethischer Perspektive systematisch zu behandeln. Vielmehr liefert er Notizen zum Weiterschreiben. Die eingenommene ethische Perspektive ist die von Menschen, die gesellschaftlich etwas bewegen wollen. Das können zivilgesellschaftliche Initiativen sein, oder auch Unternehmer/-innen, denen es um ein Mehr an Gerechtigkeit, gerade auch an globaler Gerechtigkeit geht. Denn was nützt es, daran zu appellieren, dass die Würde der menschlichen Person den Maßstab für die Digitalisierung bilden soll, wenn es keine Kräfte gibt, die dafür eintreten. Eine christlich sozialethische Perspektive ist dabei besonders herausgefordert, die Prozesse der Digitalisierung aus der Sicht der Benachteiligten und Verlierergruppen zu thematisieren.

\section{Digitalisierung - die Veränderungen kommen}

Der Begriff der Digitalisierung kann, abhängig vom jeweiligen Kontext, mehrere Bedeutungen annehmen. Im ursprünglichen Sinn meint Digitalisierung das Umwandeln von analogen In- 
formationen in digitale Formate. In der politischen Diskussion steht der Begriff für die Herausbildung einer digitalen Ökonomie aufgrund einer sich rasant weiterentwickelnden Technik. Die Digitalisierung schafft neue ökonomische Handlungsoptionen durch die Vernetzung von Sensorik, neuen Datenauswertungen, künstlicher Intelligenz, algorithmischen Entscheidungsprozessen und Mensch-Maschine-Interaktionen.

Eine weitere Bedeutung von Digitalisierung liegt in der digitalen Revolution, auch als digitaler Wandel oder digitale Transformation bezeichnet. Hier geht es um die Veränderungsprozesse in Wirtschaft, Kultur, Bildung und Politik (Gabler Wirtschaftslexikon, 2018).

In Konzepten wie Internet der Dinge, Smart Factory, Künstliche Intelligenz oder Arbeit 4.0 werden diese Veränderungsprozesse konkret.

Wer vom Internet der Dinge spricht, meint die Interaktion der realen Welt mit dem Netz der Daten. Die Gegenstände des Alltags kommunizieren über Vernetzungsstrukturen miteinander, ganz automatisch. Kontinuierlich ändern sich in der Folge Konsumverhalten und Lebensstile. Prognosen gehen davon aus, dass im Jahr 2050 über 50 Milliarden Geräte weltweit in den Sektoren Produktion, Wohnen, autonome Mobilität und Gesundheit mit dem Internet verbunden sein werden.

Die Smart Factory, also die intelligente Fabrik, ist ein zentrales Element der digitalisierten Wirtschaft. In Echtzeit tauschen die Stationen einer Produktionskette Daten miteinander aus, um in einer automatisierten Produktion dem individuellen Kundenwunsch zu entsprechen (ebd., 2018).

Die Fortschritte der Forschung zur Künstlichen Intelligenz bilden das Rückgrat der neuen Industrie- und Dienstleistungsangebote. Zahlreiche Aufgaben, die zuvor Menschen erledigt haben, können nun Algorithmen bzw. Maschinen präziser, schneller und preiswerter lösen. Bei Bosch soll Künstliche Intelligenz zu der Kernkompetenz des Unternehmens ausgebaut werden. „Wir wollen Maschinen bauen, die lernen und intelligent handeln können" (zit. n. Preuß, 2017, S. 1) lautet die Mission. Schon in zehn Jahren will der Traditionskonzern nur noch Dinge anbieten, die selbst über Künstliche Intelligenz verfügen oder zumindest mit entsprechenden Methoden hergestellt wurden. Um das Thema in alle Geschäftsbereiche hineinzutragen, wurden für die nächsten fünf Jahre 300 Millionen Euro bereitgestellt (Preuß, 2017).

Der Ausdruck Arbeit 4.0 schließlich bündelt die Konsequenzen der skizzierten Entwicklungen für die Arbeitswelt. Offen ist dabei, wie die quantitative Arbeitsplatzbilanz des digitalen Strukturwandels ausfallen wird. Arbeitsplätze verändern sich rasant, Berufe entstehen, manche verschwinden, lebenslanges Lernen wird wichtiger. Im „Grünbuch Arbeiten 4.0" zeigt sich die deutsche Bundesarbeitsministerin überzeugt: „Der schon länger anhaltende Trend zur Wissensgesellschaft wird sich auch durch die Digitalisierung fortsetzen. In der Konsequenz werden sich Wertschöpfung und Arbeitsplätze vom Produktionsbereich in den Dienstleistungsbereich verlagern. Hier arbeiten bereits heute über $73 \%$ der Erwerbstätigen “ (Kühl, 2015). Es kommt zur „Dualisierung des Arbeitsmarkts“. Dies soll bedeuten: Auf der einen Seite finden sich mehr hochqualifizierte und stark spezialisierte Facharbeiter. Auf der anderen Seite minimal bezahlte „Clickworker“ (Kühl, 2015). Das meint Internetnutzer, die nach dem Croudsourcing-Prinzip
Aufträge für Firmen erledigen, ohne in einem regulären $\mathrm{Ar}$ beitsverhältnis zu stehen.

\section{Digitalisierung - eine große Erzählung}

Diese Veränderungen sind eingebettet in eine große Erzählung, die immer weitere Kreise zieht. Sie heißt „Industrie 4.0“. Eine kleine Gruppe von Forschungs- und Industriepolitikern, von Industrievertretern und der deutschen Regierung hat 2011 im Rahmen der Hannover Messe die Formel „Industrie 4.0“ erfunden und eine breit angelegte Öffentlichkeitskampagne gestartet. Mit der Formel soll ausgedrückt werden, dass es aktuell um einen tiefgreifenden Wandel des Wirtschaftens geht, der die Produktivität der Arbeit enorm steigern wird. „Mit ,Industrie $4.0^{\prime}$ behaupten und propagieren die deutsche Industrie und die Bundesregierung eine umfassende digitale Modernisierung der industriellen Wertschöpfung. Entscheidungsträger in den Unternehmen sollen zu Investitionen in neue Maschinen, zu Softwarekäufen und der Umstellung von Produktionsverfahren ermutigt werden. Die Bürgerinnen und Bürger sollen positiv gestimmt werden, damit die Modernisierung reibungslos läuft und der Export von digitalisierten Produkten des deutschen Maschinen- und Anlagenbaus öffentlich gefördert werden können" (Emunds, 2017, S. 1).

Dabei bleibt es eine offene Frage, ob der Umbruch durch die Digitalisierung in den entwickelten Industrieländern tatsächlich sehr dramatische Folgen haben wird. Hinsichtlich der Arbeitsmarktbilanz sind sich die Experten ebenso wenig einig wie hinsichtlich der Entwicklung der Produktivität. Die Vermutung eines sprunghaften Anstiegs der Arbeitsproduktivität ist jedenfalls empirisch nicht zu bestätigen. Auch hat sich der Einsatz neuer digitaler Technologien in einigen Vorreiter-Branchen bisher noch nicht in einem starken Anstieg der Produktivität dieser Branchen niedergeschlagen. Ebenso ist die Frage, ob sich mit der Digitalisierung unsere Kulturtechniken tiefgreifend verändern, durchaus offen. Wohl scheint es so zu sein, dass sich durch Digitalisierung und Internet die Mechanismen der Wahrnehmung verändern und eine grundlegende kulturelle Veränderung herbeiführen können, diese „Hypertextstrukturen im Internet sind anders angelegt als ein geschriebener Text, eine Webseite bleibt unter ihren Möglichkeiten, wenn man sie ausdruckt. Womöglich wandelt sich unser Denken vom logisch-hierarchischen in ein flächig vernetztes. [...] Denken in Kategorien von Netzwerken statt kausal-genetischer Logik, wie sie die abendländische Tradition geprägt hat, wird zu einer neuen Form der Weltaneignung" (Rödder, 2016, S. 380).

Damit sind die Dimensionen des Wandels umrissen, den die große Erzählung von „Industrie 4.0“ umschreibt und mit der ein großes Versprechen verbunden wird.

\section{Digitalisierung - ein großes Versprechen}

Wie bei jeder neuen Entwicklungsstufe der Industrialisierung geht auch mit der vierten Stufe das Versprechen einher, dass sich die Lebensverhältnisse zum Besseren entwickeln werden. Ein neues Zeitalter mit gerechteren Arbeits- und Lebensbedingungen und einem Mehr an globaler Gerechtigkeit soll anbrechen.

Die abgehängten Regionen, national wie international, sollen durch die neuen Produktionsmethoden, durch Crowdworking, 3D-Druck etc. neue Entwicklungschancen erhalten, 
die sie nur noch ergreifen müssen. Versprochen wird eine smarte Nachhaltigkeit bei Verkehr, Klima, Wohnen und Produktion. Vor allem aber geht es um eine weitere Runde im internationalen Standortwettbewerb, bei der bisher nicht erkennbar ist, dass die weniger entwickelten Regionen in großem Maße profitieren werden.

In ihrem Bericht „2016 World Development Report on the internet - Digital Dividends" (Wilkens, 2016) warnt die Weltbank, dass die Digitalisierung nicht automatisch zu einer positiven Entwicklung führt. Zwar haben sich die technischen Möglichkeiten in den vergangenen zehn Jahren rasch verbreitet, aber nicht in dem Ausmaß wie vorhergesagt. So verdreifachte sich die Zahl der Internetnutzer auf 3,2 Milliarden, dennoch bleiben mit 4 Milliarden 60 Prozent der Menschen ohne Zugang. Etwa eine halbe Milliarde Menschen leben außerhalb eines Mobilfunknetzes. Zwar birgt die Digitalisierung, nach Ansicht des Weltbank-Ökonomen Deepak Mishra (zit. n. Wilkens, 2016), viel Potenzial, ,aber aus den Investitionen in die Technik auch alle Vorteile zu ziehen, erweise sich als schwieriger, als von Experten gedacht. Stattdessen drohten die Menschen ohne Zugang zu digitaler Technik immer mehr den Anschluss zu verlieren“ (S.1). Die Weltbank schlussfolgert, dass die „analogen Faktoren“ stimmen müssen, also die politischen und regulatorischen. Die Digitalisierung sei kein positiver Selbstläufer, sondern verstärke lediglich bereits gegebene Tendenzen, und das könne auch der Trend zu Marktkonzentrierung und Ungleichheit sein. Das gilt auch, laut den Weltbank-Ökonomen, für den Arbeitsmarkt, der sich weiter polarisieren wird. Viele Routine-Arbeiten wie zum Beispiel in Fabriken werden automatisiert, Menschen ohne ausreichende Qualifizierung drohen, in noch niedriger bezahlte Nischen des globalen Arbeitsmarkts abzurutschen. Was passiert z.B. mit den hunderttausenden Arbeiterinnen in der Textilindustrie, die Massenware herstellen, wenn Roboter nähen lernen? Adidas hat mit der Speedfactory in Ansbach, wo Schuhe vollautomatisch hergestellt werden, begonnen, die Produktion wieder nach Deutschland zurückzuverlegen. Wenn aber ganze Produktionslinien aus Ländern mit einer hohen Basisarbeitslosigkeit verschwinden, was bleibt dann vom Versprechen einer besseren Zukunft übrig?

\section{Digitalisierung - Bildung als politische Praxis}

Im Zuge der Digitalisierung wird der Ruf nach Bildung lauter, auch international. Gerade in Entwicklungsländern setzt man auf virtuelle Lernplattformen, die die Defizite der eigenen Bildungslandschaft kompensieren sollen. Bildung wird hier verstanden als Anpassungsprozess an die technische Dynamik. Selten geht es um ein Bildungsverständnis, das Menschen und Gemeinwesen befähigt, den Wandel aktiv zu gestalten. Aber auch hier ist das letzte Wort noch nicht gesprochen. So betonte Jack Mar, Vorstandvorsitzender der Alibaba Group, beim world economic forum 2018: „Alles, was wir unseren Kindern beibringen, muss sich von dem unterscheiden, was Maschinen können" (www.youtube.com/watch?v=mIbp33OA54M). Ihm zufolge können wir diesen nur mit Werten, Mitgefühl und Überzeugung überlegen bleiben. Insofern muss es ein globales ethisches Bildungsziel sein, Menschen zu befähigen, Maschinen als Subjekt gegenüberzutreten. Oder mit Kate Crawford (zit. n.
Beuth, 2016, S. 1) gesprochen: „Lernende Maschinen und Algorithmen brauchen eine Datengrundlage, die ethischen Regeln folgt. Andernfalls werden uns Maschinen in Schubladen einsortieren, in die wir weder gehören noch wollen."

Die Aufgaben für eine handlungsorientierte sozialethische Bildungsarbeit lauten dann aus meiner Sicht: aufklären erzählen - vernetzen - befähigen.

\section{„aufklären“}

Zu den großen Mythen und Erzählungen der Digitalisierung ist schon einiges gesagt worden. Bildungsarbeit hat die Aufgabe, sie zu hinterfragen. Während in Deutschland über den schleppenden Ausbau des Breitbandnetzes geklagt wird, schaffen Unternehmen wie Amazon, Apple, Uber etc. Fakten. In nur zehn Jahren sind sie zu den gewinnträchtigsten Unternehmen der Welt aufgestiegen und mit Hilfe der Welthandelsorganisation bauen sie diese Position aus. Das ist nur ein Beispiel von Entwicklungen und Zusammenhängen, auf die eine handlungsorientierte sozialethische Bildungsarbeit in globaler Perspektive eingehen muss. Sie benötigt dazu die entsprechende Expertise. Leider steht die wissenschaftliche Aufarbeitung noch am Anfang. Internationale Hilfsorganisationen können hier mehr investieren, um zu einer komplementären Erzählung beizutragen, die den Ambivalenzen gerecht wird. Einen Schritt in diese Richtung hat „Brot für die Welt“ mit der kleinen Studie von Sven Hilbig (2017) „Auswirkungen der Digitalisierung auf den globalen Süden“ getan. Dort heißt es: „In Anbetracht der bestehenden Risiken, welche die Digitalisierung für die Länder des globalen Südens mit sich bringt, ist es an der Zeit, sich aus entwicklungspolitischer Perspektive kritisch mit den verschiedenen Facetten dieser technischen Revolution zu beschäftigen. Angefangen bei dem Versuch der Hightech-Konzerne, die Regelungen des Welthandels nach ihren Interessen zu stricken bis hin zu der Frage, welche politischen, gesellschaftlichen und ökonomischen Rahmenbedingungen gegeben sein müssten, damit die Digitalisierung tatsächlich einen Beitrag zur Umsetzung der Agenda 2030 leisten kann. Einer Agenda, der gegenüber die gesamte Staatengemeinschaft verpflichtet ist" (Hilbig, 2017, S. 9).

\section{„vernetzen“"}

Vernetzung ist das Schlagwort des Digitalisierungsprozesses. Sie bildet eine globale „Netzwerkgesellschaft“ (Castells), mit einer Weltwirtschaft als Netzwerk von Märkten, Finanztransaktionen und Arbeitskräftepools, den „Netzwerkunternehmen“ als neuer Form der Geschäftsorganisation sowie der internationalen Vernetzung sozialer Bewegungen über das Internet (Rödder, 2016, S. 30). Auch dient der Netzwerkbegriff als „Metapher zur Beschreibung neuer Sozialbeziehungen im digitalen Zeitalter" (ebd., S. 31).

Eine handlungsorientierte sozialethische Bildungsarbeit kann diese „neue“ Sozialität fördern und gestalten. Dabei sind die Werte Solidarität und Teilhabe unter den veränderten Rahmenbedingungen neu in den Blick zu nehmen. Die Digitalisierung schafft neue Möglichkeiten der Solidarisierung. In Echtzeit können Protest und Unterstützung organisiert werden. Aber Solidarität ist auch durch die mit der Digitalisierung einhergehende Ausdifferenzierung der Öffentlichkeit bedroht. Soziale Netzwerke, auf Teilgruppen bezogene Nachrichtenange- 
bote und Suchmaschinen fragmentieren die Wahrnehmung der gesellschaftlichen Wirklichkeit. Zudem sind Beteiligungschancen, Einkommensmöglichkeiten, aber auch Freiheitspotentiale immer mehr durch den Zugang zum Internet geprägt. Dieser Zugang aber ist sehr unterschiedlich verteilt. Weltweit sind mehr Menschen offline als online. Die Grenze verläuft dabei national wie international entlang der Wohlstandsgefälle und verschärft so bestehende Ungleichheiten. Das gilt auch hinsichtlich einer Medienkompetenz. Diese ist mehr denn je eine Kulturtechnik, die „für die Teilhabe am politischen Geschehen wie überhaupt für die Wahrnehmung von Bürgerrollen in einem modernen demokratischen Gemeinwesen unverzichtbar ist" (Sarcinelli, 2011, S. 27). Im schlimmsten Fall verstärkt eine ungleiche Medienkompetenz bestehende Ungleichheiten, sie schwächt die Schwachen und stärkt die Starken. „Wenn die Potentiale des Internets demgegenüber zu mehr Freiheitsspielräumen in allen Teilen der Gesellschaft führen sollen, dann müssen digitales Lernen und eine damit verbundene Medienkompetenz Eingang in die schulische und politische Bildungsarbeit finden" (Kerst, 2015, S. 2).

\section{„erzählen“}

Kommunikation gilt als eines der weiteren großen Worte im Digitalisierungsprozess. Deutet man es im Sinn einer handlungsorientierten sozialethischen Bildungsarbeit, dann besteht der Wert von Kommunikation im Umbruchsprozess der Digitalisierung darin, die Geschichten der Betroffenen öffentlich zu erzählen. Wenn als sicher geglaubte Orientierungen zerbrechen, besteht die Notwendigkeit einer neuen Verständigung. Das gilt sowohl für Prozesse in den Betrieben wie auch im gesellschaftlichen und globalen Kontext. Im Mittelpunkt steht dabei für die Betroffenen der Umbruch in ihrem unmittelbaren Lebensraum. Insbesondere im Umbruch der Arbeitswelt liegt ein Ansatzpunkt für einen erzählenden Zugang.

Arbeit strukturiert die entwickelten Industriegesellschaften. Dem Vorrang des Arbeitsprozesses sind Unterbrechungen wie Elternzeit, Krankschreibungen etc. zugeordnet und selbst wer arbeitslos ist, definiert sich über das Fehlen von Arbeit. Arbeit strukturiert den Sozialstaat. Weil Arbeit ein so zentrales Moment ist, muss gerade in Umbruchszeiten über ihr Wesen, ihren Sinn und über ihre Funktion für die Gesellschaft nachgedacht werden.

Die Medien haben dies entdeckt und seit einigen Jahren gibt es in Rundfunk, Fernsehen und Blogs regelmäßig Serien zum Thema „Zukunft der Arbeit“. Hier kommen Beschäftigte und Experten gleichermaßen zu Wort. Besonders interessant ist eine Aktion des Arbeits- und Sozialministeriums in den Niederlanden unter dem Titel: Wie arbeiten die Niederlande? (Ministerie van Sociale Zaken en Werkgelegenheid, 2018 )

In dieser Richtung sollte eine handlungsorientierte sozialethische Bildungsarbeit weiterdenken. Ein Schwerpunkt könnte es sein, die internationalen Geschichten zu heben und Betroffene ins Gespräch zu bringen, denn die internationale Perspektive ist bisher unterbelichtet. So könnte es gelingen, in der globalen Umbruchssituation konkrete oder auch virtuelle Erzählgemeinschaften zu konstituieren, mit dem Ziel, der einen großen Erzählung eine Vielfalt von Erzählungen an die Seite zu stellen, um zu verdeutlichen, dass gesellschaftliche Entwicklungen nie alternativlos sind. „befähigen“"
Digitale Ethik kommt oft in Manifesten oder Geboten daher. Sie tragen die Lehre über das „Gute“ vor, formulieren Appelle und moralische Forderungen. Diese Ethik befähigt nicht zum Handeln, sondern adressiert ihre Empfehlungen an Politik, Wirtschaft oder den Einzelnen. Eine handlungsorientierte sozialethische Bildungsarbeit dagegen reflektiert und initiiert im unmittelbaren Kontext handelnder Akteure. Inspirierend für diesen Zugang sind immer noch die Arbeiten des brasilianischen Pädagogen Paolo Freire, so jedenfalls sieht es Michael Schwinger (2005). Freire will mit seiner Pädagogik durch Bewusstseinsbildung und konkretes Tun die Autonomie des Menschen fördern. Seine Arbeiten haben nicht nur bei Fragen der Bürgerpartizipation und Gestaltung der Zivilgesellschaft, sondern auch in der Begleitung des technologischen Wandels eine hohe Relevanz. Denn, wer sind diejenigen, die im Rahmen dieser Pädagogik ethische Kriterien entwickeln und handeln? Es sind die Betroffenen der Digitalisierung. Den Prozess aus ihrer Perspektive zu gestalten, scheint die Aufgabe der Stunde zu sein.

So sehen es auch viele Experten und Protagonisten des technologischen Wandels, denn weltweit kann dieser zum Stillstand kommen, wenn die Menschen nicht mehr gestaltende Subjekte, sondern bloße Opfer der Entwicklung sind. Längst häufen sich die Anzeichen, dass der Höhepunkt der Digitalisierung bald erreicht sein wird und sich die Technologisierung der Gesellschaft auf ein erträgliches Maß einpendeln könnte. „Ausgerechnet im Herzen von Hightech-Land, San Francisco, begehrt eine Anti-Tech-Bewegung (darunter eine anarchistische Gruppe namens „The Counterforce“) gegen IT-Riesen wie Google auf und auch bei den Nutzern neuer Technologien kommt es zu „zarten digitalen Ermüdungserscheinungen“ (Steinschaden, 2014, S.1). Der Boom von Facebook ist schon länger vorbei. Dazu kommt der Wunsch nach mehr digitaler Privatheit, der User von Facebook und Twitter in Richtung WhatsApp, Snapchat, Whisper oder Secret zieht, wo es nicht mehr um das Sharen mit der Weltöffentlichkeit, sondern um vertrauliche Gruppen-Chats und Anonymität geht. Vielleicht sind diese Smartphone-Apps sogar ein Zwischenschritt zurück in analoge Gefilde und entwöhnen uns langsam vom „Always on"-Paradigma (Steinschaden, 2014, S. 1).

Die massive Zunahme von Burn-out, der Verschreibung von Psychopharmaka und leistungssteigernden Mitteln, pandemisches Mobbing sind eine Realität der digitaler werdenden Gesellschaften, für die sich der Begriff der „Dopinggesellschaften" (Väth, 2016, S. 63) etabliert hat. In einigen Ländern beginnt der Gesetzgeber gegenzusteuern, so z.B. in Frankreich. Dort wurde im August vergangenen Jahres im Arbeitsgesetzbuch ein Recht des Arbeitnehmers, offline zu sein, festgeschrieben.

Den westlichen Ländern dürfte es gelingen, aufgrund ihres Reichtums und funktionierender Strukturen, Konsequenzen für die Sozial-, Arbeitsmarkt- und Gesundheitspolitik durchzusetzen. So hat hier eine Debatte um das bedingungslose Grundeinkommen begonnen, das neue Freiheitsräume in der Arbeits- und Lebenswelt schaffen soll, wenn der Roboter als Steuerzahler einspringt. Auch gibt es in den Ländern zivilgesellschaftliche Bewegungen wie die "Gemeinwohlökonomie“, „New Work“, oder die „offliner“, die neue Leitbilder des Wirt- 
schaftens und Arbeitens entwickeln. Diese Orte mitzugestalten und sie in globaler Perspektive zu weiten sollte die Aufgabe ethischer Reflexion und Praxis sein.

\section{Schluss}

Von Globalisierung und Digitalisierung wird oft so gesprochen, als müssten die Nebenwirkungen um jeden Preis in Kauf genommen werden. Der Wirtschaftsethiker Thorsten Busch (2017) regt an, diese Sprache in Form von Heilsversprechen zu hinterfragen und die Nutzen und Risiken für das Individuum in den Mittelpunkt zu stellen. Wer dies im Kontext einer handlungsorientierten sozialethischen Bildungsarbeit leistet, stellt fest, dass die Gegentrends stärker werden. „Achtsamkeit ist ganz klar der größte Gegentrend zur Digitalisierung “, erklärt Harry Gatterer (zit. n. Griese, 2017), Geschäftsführer des „Zukunftsinstituts". Seiner Ansicht nach zeichnet sich ein echter Mindshift ab, der zu einer neuen Achtsamkeitsqualität in der Wirtschaft und zu völlig neuen Konsumwelten führen wird. So identifizieren die Forscher/innen des Instituts eine ,junge, stark vernetzte und gut ausgebildete Generation Global“" (www.zukunftsinstitut.de/artikel/die-neue-achtsamkeit) die nach einem achtsamen Umgang mit Umwelt und Mitmenschen strebt und, geprägt von Sinnsuche und ethischer Überzeugung, hohe Anforderungen an die Arbeitswelt stellt, abseits vom alten „schneller, höher, weiter". Diese Entwicklung zu unterstützen, zu begleiten, um entwicklungspolitische Aspekte anzureichern und konkrete Aktionen zu initiieren ist Aufgabe einer handlungsorientierten sozialethischen Bildungsarbeit. Holger Volland, Autor und Vize-Präsident der Frankfurter Buchmesse, bemerkt, dass „Künstliche Intelligenz eine wichtigere Rolle spielen wird als etwa Gentechnologie oder Atomkraft. Mit beiden Themen haben wir uns sehr intensiv auseinandergesetzt, das sollten wir mit künstlicher Intelligenz auch tun" (Volland, 2018). Die gesellschaftliche Auseinandersetzung um Atomkraft und Gentechnologie hat Gegenbewegungen hervorgebracht. Daran gilt es zu erinnern. Es hat viele Jahre gedauert, bis die Anti-Atomkraft-Bewegung - oder andere, wie die Biooder fair trade-Bewegung, gesellschaftlich und ökonomisch wirksam Einfluss nehmen konnten. Womöglich fehlt angesichts der technischen Beschleunigung durch die Digitalisierung hierzu die Zeit. Womöglich beschleunigt die Digitalisierung aber auch die Bildung von Gegentrends. Wie auch immer - die Entwicklung ist nicht ohne Alternativen.

\section{Literatur}

Al-Ani, A. (2017). Industrie 4.0. in der Peripherie. Zugriff am 15.03.2017 www.zeit. de/karriere/2017-01/digitalisierung-entwicklungslaender-armut-arbeitsmarkt

Beuth, P. (2016). Re:publika. Ein Königreich für eine KI-Ethik. Zugriff am 15.03.2018 www.zeit.de/digital/internet/2016-05/kuenstliche-intelligenz-republicaethik-kate-crawford
Busch, T. (2017). Ethik in der Digitalisierung. Technik ist niemals neutral. Zugriff am 15.3.2017 www.swisscom.ch/de/business/enterprise/themen/digital-business/ethikin-der-digitalisierung.html

Capurro, R. (2015). Digitale Ethik. Bussiness impact (4)2015.Zugriff am 15.03.2018 www.capurro.de/businessimpact.html

Castells, Manuel (2003). Das Informationszeitalter. Bd.1: Der Auftieg der Netzwerkgesellschaft. Opladen: Leske+Budrich.

D21 (2017). Denkimpuls Digitale Ethik: Grundlagen der Ethik - Eine normative Orientierung in der vernetzten Welt. Zugriff am 15.03.2018 https://initiatived21.de/ publikationen/denkimpulse-zur-digitalen-ethik/

Emunds, B. (2017). Digitalisiert arbeiten - gerecht Wirtschaften. Symposium zur KSI-Eröffnung am 6. Mai 2017. In Oswald-von-Nell-Breuning-Institut (Hrsg.), Frankfurter Arbeitspapiere. Frankfurt.

Gabler Wirtschaftslexikon online (2018). Digitalisierung. Zugriff am 15.03.2018 www.wirtschaftslexikon.gabler.de/Definition/digitalisierung.html

Griese, I. (2017). Achtsamkeit ist der Gegentrend zur Digitalisierung. Zugriff am 18.03.2018 www.welt.de/icon/partnerschaft/article164369793/Achtsamkeit-istder-Gegentrend-zur-Digitalisierung.html

Hilbig, S. (2017). Auswirkung der Digitalisierung auf den globalen Süden. Zugriff am 15.03.2017 www.info.brot-fuer-die-welt.de/blog/digitalisierung-birgt-risikenglobalen-sueden

Kerst, V. (2015). Freiheit, Gerechtigkeit und Solidarität in Zeiten der Digitalisierung. Zugriff 03.03.2018 http://www.forumnetzpolitik.de/2015/03/18/freiheit-gerech tigkeit-und-solidaritaet-in-zeiten-der-digitalisierung

Kühl, S. (2015). Vision des Arbeitsministeriums. Alles so vernetzt hier. Zugriff am 10.03.2018 www.faz.net/aktuell/feuilleton/debatten/gruenbuch-des-arbeitsminis teriums-arbeit-4-0-13815910.htm

Mar, Jack; Vorstandvorsitzender der Alibaba Group (2018): world economic forum. Zugriff am 01.10.2018 www.youtube.com/watch?v=mIbp33OA54M)

Ministerie van Sociale Zaken en Werkgelegenheid (2018). Hoe werkt Nederland. Zugriff am 18.07.2018 www.hoewerktnederland.nl

Preuß, S. (2017). Künstliche Intelligenz ist die Zukunft von Bosch. Zugriff am 25.03.2018 www.faz.net/aktuell/wirtschaft/bosch-investiert-millionen-in-kuenst liche-intelligenz-15156050.html

Rödder, A. (2016). 21.0. Eine kurze Geschichte der Gegenwart. München: Ullstein. https://doi.org/10.17104/9783406682476

Sarcinelli, Ulrich (2011). „Medien und Demokratie“. Demokratie in Deutschland 2011 - Ein Report der Friedrich-Ebert-Stiftung. Zugriff am 01.10.2018 www.demokratiedeutschland-2011.de/common/pdf/Medien_und_Demokratie.pdf

Schwinger, M. (2005). Medieneinsatz als Instrument pädagogischer Arbeit mit Straßenkindern in Brasilien. Zugriff am 03.04.2018 https://fsf.de/data/hefte/ausgabe 132/schwinger68_tvd32.pdf

Steinschaden, J. (2014). Generation Analog. Stößt die Digiatlisierung an ihre Grenzen? Zugriff am 28.07.2018 www.netzpiloten.de/generation-analog-stoesst-die-digitali sierung-ihre-grenzen/

Väth, M. (2016). Arbeit. Die schönste Nebensache der Welt. Wie New Work unsere Arbeitswelt revolutioniert. Offenbach: Gabler.

Volland, H. (2018). Wenn Maschinen Kreativ werden. Zugriff am 20.03.2018 www. detektor.fm/digital/holger-volland-ueber-kreative-kuenstliche-intelligenz

Wilkens, A. (2016). 4 Milliarden Menschen sind offline. Zugriff am 15.03.2018 www. heise.de/newsticker/meldung/Weltbank-4-Milliarden-Menschen-sind-offline3070631.html

Zukunftsinstitut (2018). Die neue Achtsamkeit. Zugriff am 01.10.2018 www. zukunftsinstitut.de/artikel/die-neue-achtsamkeit

\section{Dr. phil. Manfred Körber}

Geschäftsführer und Leiter des Nell-Breuning-Hauses in Herzogenrath, einer Tagungsstätte der politischen und sozialethischen Bildungsarbeit, zuvor 16 Jahre Abteilungsleiter im Generalvikariat des Bistums Aachen mit der Zuständigkeit u.a. für gesellschaftliche und weltkirchliche Aufgaben. 